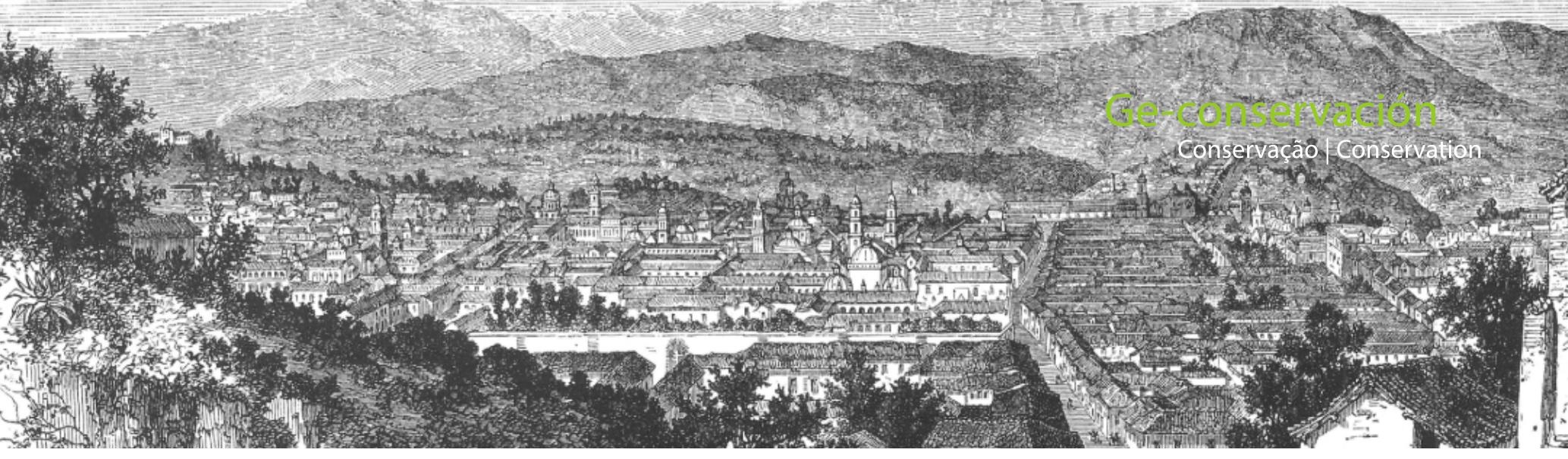

\title{
Torre de la iglesia de la Compañía de Jesús de Quito: historia, proporciones y medidas
}

\author{
Inés del Pino Martínez, M. Lenin Lara Calderón
}

Resumen: Las torres de las iglesias de Quito sufrieron daños significativos con los terremotos de 1797, 1859 y 1868, en particular la de la iglesia de la Compañía de Jesús, que según los escritos de viajeros y científicos fue la más alta y bella de la ciudad. Con el último evento fue parcialmente demolida hasta los 23,40 metros. La revisión de fuentes históricas y planos arquitectónicos realizados entre 1992 y 2019 por varias instituciones llevó a estudiar las proporciones de la fachada en relación con la torre para analizar, con métodos precisos, la altura que debió tener antes del terremoto de 1868. Los resultados de este análisis histórico, gráfico y geométrico establecen que la altura de la torre fue de 41,60 metros; existe correspondencia de proporciones y medidas entre la fachada, la iglesia, y la torre; ésta última representa la repetición de 5,5 veces el módulo base identificado en el estudio.

Palabras clave: arquitectura religiosa latinoamericana, Quito, iglesia de la Compañía de Jesús, torre, terremoto, geometría

\section{Tower of Jesuitic Church in Quito: history, proportions and measurements}

Abstract: The towers of the churches of Quito suffered significant damage from the earthquakes of 1797, 1859, and 1868, particularly that of the Compañía de Jesús church, which according to the writings of travelers and scientists was the tallest and beautiful in the city. With the last event it was partially demolished to 23.40 meters. The review of historical descriptions and architectural plans made between 1992 and 2019 by various institutions led to the study of the proportions of the façade in the relationship to the tower to analyze with precise methods, the height it must have had before the 1868 earthquake. The results of the historical, graphic, and geometric analysis established that the height of the tower was 41.60 meters; there is correspondence of proportions and measures between the facade, the church, and the tower, the tower represents 5.5 times the base module identified in the study.

Keywords: Latin American religious architecture, Quito, Jesuit church, tower, earthquake, geometry

\section{Torre da igreja da Companhia de Jesús de Quito: história, proporções e medidas}

Resumo: As torres das igrejas de Quito sofreram danos significativos com os terramotos de 1797, 1859 e 1868, em particular a da igreja da Companhia de Jesus, que segundo os escritos de viajantes e cientistas foi a mais alta e bela da cidade. Com o último evento foi parcialmente demolida até aos 23,40 metros. A revisão de fontes históricas e planos arquitetónicos realizados entre 1992 e 2019 por várias instituições levou a estudar as proporções da fachada em relação à torre para analisar, com métodos precisos, a altura que deveria ter tido antes do terramoto de 1868. Os resultados desta análise histórica, gráfica e geométrica estabelecem que a altura da torre foi de 41,60 metros; existe correspondência de proporções e medidas entre a fachada, a igreja, e a torre; esta última representa a repetição de 5,5 vezes o módulo base identificado no estudo.

Palavras-chave: arquitetura religiosa latino-americana, Quito, igreja da Companhia de Jesus, torre, terramoto, geometria 


\section{Introducción}

Un ejemplo relevante de arquitectura barroca en Latinoamérica es la iglesia de la Compañía de Jesús de Quito por la calidad del arte y la solidez de su arquitectura. El edificio, compuesto por la iglesia, la universidad, el colegio y espacios domésticos, se construyó en un periodo de 160 años: entre 1605 y 1765. Con la expulsión de los jesuitas en 1767, los espacios se abrieron por temporadas cortas, mientras pasaron a cargo de comunidades religiosas o de entidades civiles. A su retorno en 1851 la organización encontró sus edificios deteriorados, no solo por la falta de mantenimiento sino por el efecto de terremotos, en particular el de 1797 y el sismo de 1859, éste último cuarteó la torre, y aunque tuvo una reparación inmediata; apenas restablecida, se volvió a cuartear con el terremoto de 1868, razón por la que se ordenó derribarla a la mitad de su altura.

El estudio del impacto de los terremotos en la arquitectura colonial de Quito demuestra que los elementos arquitectónicos más afectados fueron las torres (Del Pino 1990: 67-100). El periodo entre 1859 y 1868 se lo podría denominar "entre dos terremotos" por las complejas circunstancias de orden político y técnico que rodea a un ciclo de destrucción, reconstrucción, cuarteamiento y demolición parcial de la torre más alta de la ciudad. Las particularidades de orden político que coinciden en este tiempo son: acciones del presidente Gabriel García Moreno, quien posibilitó el retorno de los jesuitas y el establecimiento de una reforma educativa con el apoyo de científicos alemanes en favor de la formación técnica, todo esto impacta en la manera de percibir el nuevo orden republicano. Las particularidades de orden técnico se relacionan con los esfuerzos de los jesuitas retornados por reconstruir su edificio, restituir la torre, y recuperar sus bienes, y por otra parte, la voluntad política del propio García Moreno quien hizo tomar las medidas para reconstruirla "en su estado anterior" a expensas de su propio peculio (Jouanen S.J. 2003: 1-39).
Los terremotos de 1859 y 1868 sumaron nuevos daños estructurales en los muros de los edificios, los que no siempre fueron reparados de inmediato por razones económicas, o descuido de las autoridades de turno; ante la emergencia, los fondos disponibles se utilizaron en arreglos indispensables para mantener en pie las mamposterías o demoler lo que quedó en pie. Por esta razón, la secuencia de fotografías, dibujos y descripciones de la época exteriorizan torres distintas en menos de un siglo.

La adaptación a la naturaleza geológica de Quito llevó consigo el acomodo de las edificaciones y a tomar decisiones sobre la composición volumétrica, es decir, dimensión modesta y materialidad adecuada para que la fábrica soporte nuevos embates de la naturaleza, destacando en las fachadas de las iglesias las portadas talladas en piedra, interiores decorados con pinturas y relieves, superficies de madera policromada y dorada; mientras tanto, el interior de los claustros es austero, allí, el valor de la arquitectura está expresado en el espacio. El carácter concedido a Quito de "gran convento", atribuida a Simón Bolívar, tiene relación con la cantidad y calidad de edificios construidos durante la colonia, en donde en apenas 54 hectáreas se concentró la representatividad de la iglesia católica mediante 17 iglesias, obras monumentales en la que constructores y arquitectos sugirieron un orden clásico y barroco, pensado y ajustado a las circunstancias topográficas andinas en la escala de un damero más o menos regular.

Las iglesias coloniales de Quito tienen una o dos torres que son elementos arquitectónicos verticales presentes en la fachada; están construidas en ladrillo; destacan en el paisaje para convertirse en hitos urbanos de orientación e identificación de cada orden religiosa e iglesia en la ciudad; en conjunto, delinean el perfil urbano [Figura 1]; la torre evidencia el poder de la iglesia; el sonido de las campanas marcó el tiempo en los habitantes de Quito,

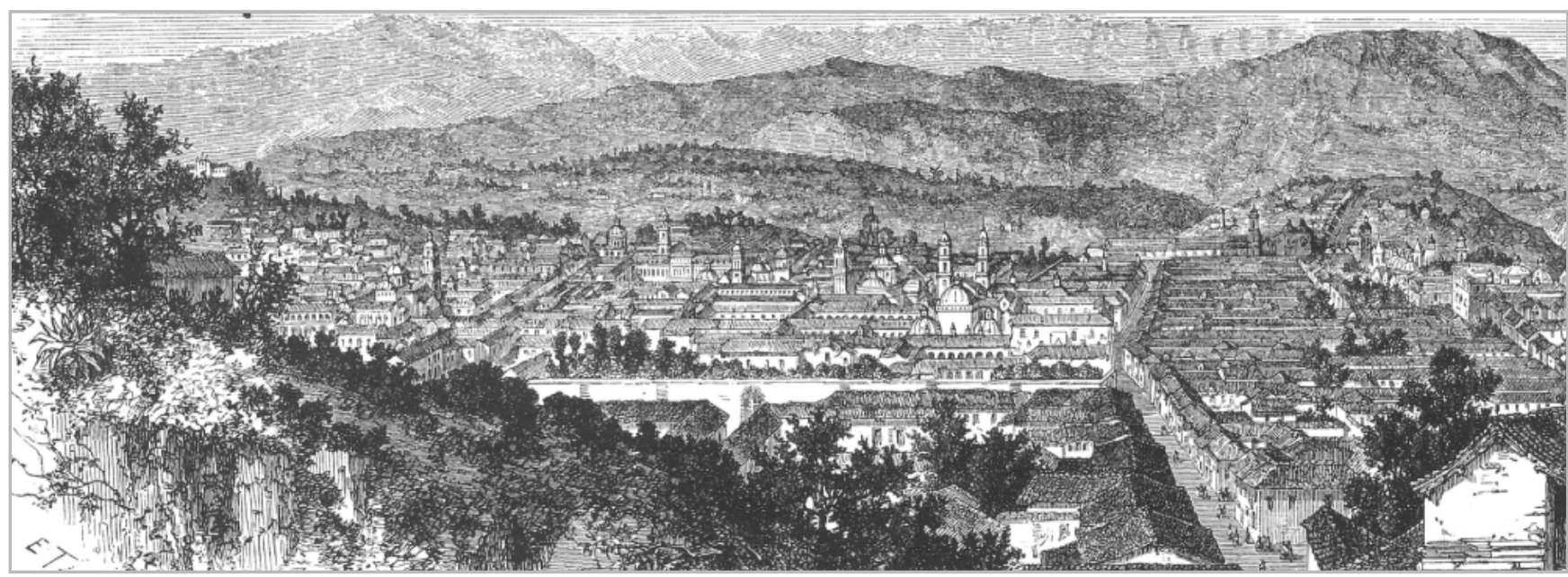

Figura 1.- Vista general de Quito. Dessin E. Therond d'après M. Ernest Charton. En Le Tour du Monde, 1867: 407 
es decir, la cotidianidad: la hora de la misa, la muerte de alguien, la alerta pública, e inclusive, la convocatoria a la revuelta y la protesta social.

La fundación española de Quito en 1534 se produjo en un espacio de difícil acceso, asentada al pie del volcán Pichincha, entre tres colinas y atravesada por tres quebradas profundas que cortaban la ciudad de oeste a este. El 8 de enero de 1610 el cabildo ordenó la construcción de varios puentes en la ciudad, el mismo año se rellenó la quebrada que pasaba junto a la Catedral, lo que sugiere un programa de consolidación y expansión urbana (Herrera 1916: 73). Sin embargo, el plano de Quito de Gualberto Pérez de 1888 revela que el relleno se redujo a los cruces de las calles principales para evitar hacer puentes, mientras que al interior de los predios las quebradas estaban abiertas.

Los documentos del cabildo dan cuenta que construir un puente era una obra costosa, que se financiaba con mingas de indios y mestizos, a veces obligados; $y$ aportes económicos de la gente pudiente de la ciudad a cambio de poner su nombre o recibir favores acordados con los donantes ${ }^{[1]}$. El puente formaba parte de la historia cotidiana y era necesario para la expansión de la ciudad; aunque la relación entre barrios no cambió significativamente. De las tres quebradas que cruzaron la ciudad de oeste a este con diferentes nombres según sus tramos, la de Zanguña atraviesa el predio jesuita y la iglesia de El Sagrario. La cercanía de las edificaciones religiosas y casas particulares a las quebradas provocó la presencia de humedades, el hundimiento de algunas y contribuyó al deterioro de las edificaciones, todo esto unido al efecto de los sismos y erupciones.

Otro aspecto que precede al análisis de la proporción y altura de la torre de la iglesia de la Compañía de Jesús de Quito es la transformación reiterada de otras torres a causa de los terremotos, sismos y erupciones, lo que lleva a confirmar que la transformación de la torre de La Compañía no es la única y las que hoy estamos habituados a mirar no son las originales, sino que algunas corresponden a la segunda o tercera reconstrucción realizada hasta el siglo XX.

\section{Instrumentos del análisis}

En este apartado, el análisis cualitativo se centra en el proceso de la construcción del edificio y su relación con los terremotos. Para José Gabriel Navarro, Diego Santander y José Luis Micó S.J. (Ortiz 2008: 323), la Compañía de Jesús se construyó en tres etapas, que al ser confrontadas con el proceso constructivo mencionado por José Jouanen S.J., confirman que la primera etapa va de 1606 a 1636, en la que la iglesia y la torre se construyeron en el patio contiguo a la iglesia actual, es decir se trata de un edificio que hoy se desconoce. Según los autores, la primera torre tuvo siete metros, estuvo probablemente en el mismo lugar y la iglesia estaba concluida y habilitada para la liturgia católica.
La segunda fase, entre 1636 y 1689, arranca con la llegada de Marcos Guerra S.J., quien implantó el sistema de bóvedas de ladrillo para cubrir la iglesia y cerrar la quebrada de Zanguña, que atravesaba por la mitad del predio; en este periodo se puede inferir que las erupciones del volcán Pichincha, del 27 de octubre y del 3 de noviembre de 1660, debieron causar algún daño sobre la construcción en proceso, sobre todo la del 27 de octubre, en que la caída de ceniza oscureció la ciudad y se sintieron varios sismos. Los testimonios referidos a daños ocurridos en La Compañía son escasos, y en la ciudad, el más relevante corresponde a la descripción que se encuentra en la fachada de la iglesia de San Agustín, sobre el arco de ingreso, labrada en la piedra. Sobre La Compañía no hay una descripción específica y lo más probable es que cualquier daño fue corregido en el mismo proceso de la obra.

La carta Annua (1642 - 1652) señala que la iglesia estaba terminada y también la torre. Según el investigador Alfonso Ortiz, no fueron dos iglesias sino la misma que hoy conocemos; a su llegada, Marcos Guerra cambió el artesonado de madera por una bóveda de cañón corrido con arcos de refuerzos en su recorrido. Sobre la torre el documento señala "Acabose también la torre ... de cincuenta y cinco varas de alto [46,20 m], obra tan fuerte que sin la lesión que han padecido menores edificios ha podido resistir algunos temblores grandes" [2]. Queda la pregunta de si lo hizo por renovar la cubierta de la iglesia o a causa de daños similares a los encontrados en otros edificios. Según estimaciones del Instituto Geofísico de la Escuela Politécnica Nacional, la capa de ceniza que cubrió la ciudad fue de $30 \mathrm{~cm}$. aproximadamente, con un peso significativo como para que los techos de madera y teja cedan; con esta erupción la torre debió sufrir en menor grado por su tamaño e inclinación de la cubierta, pero no es posible comprobarlo. El cambio de techo de artesón a bóveda demanda una serie de ajustes en la estructura existente que por el momento no es evidente; no obstante, los arcos fajones de la bóveda están localizados en promedio de 7,24 m. que es posible en la modulación de un artesonado. Un análisis in situ confirmaría posibles alteraciones constructivas, así como los vestigios de cimientos de una primera iglesia. Otra pregunta por resolver es por qué la Compañía es la única iglesia en Quito con una torre detrás de la fachada principal y exenta del templo.

La tercera fase, entre 1689 a 1765, comprende la terminación de la fachada de piedra de la iglesia, entre 1723 y 1765 . La iglesia, claustros del colegio y universidad debieron sufrir algún daño entre 1751 y 1755 en que se registran dos terremotos en Quito. A manera de comparación, el terremoto de 1751 tuvo una intensidad de VII MSK, el convento de Santo Domingo sufrió daños en la iglesia y la caída de una campana; por testimonio de Bernardo Recio, la catedral quedó inservible, razón por la que posteriormente fue reforzada con barras de hierro y la torre zunchada con cadenas. Otro dato que da cuenta de la magnitud de este evento es el levantamiento de la 
clausura de seis conventos para que los religiosos puedan socorrer y transportar a heridos y muertos, y acompañar a moribundos (Recio S.J. 1947: 382).

La intensidad del terremoto de 1755 está estimada en VIII MSK, es conocido como el "terremoto de Quito" dado que su origen fue la activación de la falla geológica de Quito y esta ciudad fue la más afectada con una destrucción generalizada, tanto en construcciones civiles de tierra cruda y basamentos de piedra, como en templos religiosos construidos en ladrillo y piedra. En este evento, varias torres, entre ellas la de la Compañía (Del Pino 1990: 80-81) proporciona testimonios como el del padre Recio, quien señala que la bóveda de su aposento soportó el terremoto por haber sido "fortificada con barras de hierro desde el pasado terremoto", es decir, en 1751 (Recio S.J. 1947: 385), lo que da a conocer el tipo de soluciones adoptadas en ese entonces.

Al regreso de los jesuitas en 1850, la organización comenzó el proceso de arreglo de la iglesia y recuperación de sus bienes. En 1852 fueron expulsados nuevamente hasta 1862, en que se establecieron definitivamente en Quito. Los mayores daños de la iglesia fueron la humedad y excavaciones clandestinas en los muros exteriores. En este proceso sobrevino un terremoto en 1859 que agravó los daños ya existentes por la falta de mantenimiento. Según Jouanen, cuando el arzobispo de Quito hizo la entrega de los bienes de la iglesia y los claustros, el templo se encontraba muy deteriorado: faltaban muebles, casi todas las ventanas carecían de cristales. En el templo, las bóvedas estaban rajadas, traspasadas por el agua y la humedad, y la descripción continúa: "El artesón hundido y resquebrajado por todas partes, el pavimento desenladrillado, ...los altares vencidos y podridos por el pie" (Ortiz 2008: 203204).

Según la misma fuente:

"La torre de las campanas, ...se halla cuarteada y despedazada: es un cuadro de paredes sin gradas para subir, un campanario sin campanas, un esqueleto que tiene figura de torre, pero sin poder servir para ese objeto ni otro alguno; es un enemigo doméstico que constantemente amenaza desplomarse sobre los edificios contiguos, incluso la universidad" (Ortiz 2008: 203-204).

La reparación de la torre de la Compañía fue inmediata, para José Gabriel Navarro la torre fue "recompuesta", y más adelante señala que el terremoto de 1868 "la cuarteó tanto que no hubo más que deshacerla hasta la altura de su hermosa barbacana". José Jouanen describe que "fue preciso derribarla" para que no cayera sobre los edificios colindantes.

El terremoto de Ibarra de 1868 afectó a una amplia zona del norte de Ecuador y en particular a Quito; la descripción de Joseph Kolberg señala que la mayoría de las torres quedaron "cercenadas en una tercera parte de su medida anterior a consecuencia de los terremotos" (Kolberg 1996: 479). El 19 de abril de 1869 Thomas Reed, arquitecto de la República y un grupo de técnicos realizaron una inspección de las torres destruidas de Quito para: "tener conocimiento del estado en que se encuentren ... para dictar sus providencias a fin de precaver los males que resultarían a la población si se encontrasen ruinosas; ... disponer su demolición ... i evitar resultados perjudiciales a los habitantes" (Saldarriaga 2006: 119).

Finalmente, el Periódico Oficial de Quito del 19 de agosto de 1868 señala:

“El colegio é iglesia de la Compañía se hallan en inminente peligro, porque la torre que sobresale en medio de ellos está en riesgo de caerse. Esta torre, como las demás de Quito, se encuentran descuartizadas unas y derrumbándose otras... amenazando muerte á su alrededor, si sobreviniese otro fuerte movimiento... De paso, hay que notarse, que los templos que han sufrido más son los embovedados; prueba que esta clase de construcción no es conveniente para un país de temblores" (El Nacional (a) 1868).

En ediciones posteriores la misma fuente menciona: "De Quito se dice que, además de las cinco iglesias que se desplomaron, todas las casas han quedado en estado de ruina y que la población vive en el ejido" (El Nacional (b), 1868). Estos testimonios, junto al de la carta Annua antes citada, son argumentos para identificar y comparar la altura de las torres de Quito y al mismo tiempo confirmar si estos elementos arquitectónicos mantuvieron su volumetría y proporciones con relación a las iglesias a lo largo de tres siglos, pese a los terremotos.

\section{Geometría de la torre de la Compañía}

La forma y proporción de las torres de Quito es visible en dibujos, grabados y fotografías del siglo XIX, realizadas con la llegada de viajeros y científicos, luego de 1855, quienes posteriormente publicaron los resultados de su travesía por América. Para este artículo se tomarán dos ejemplos, un grabado de la revista "Le Tour du monde", ilustrada e impresa por Edouard Charton [Figura 2], y una fotografía de autor anónimo, que presenta la torre zunchada con bandas metálicas [Figura 3].

Estas evidencias permiten clarificar algunas descripciones como la del jesuita Juan Domingo Coleti (1727-1798) quien desde el exilio recuerda: "Se alzan algunas torres, entre las cuales la más alta y fuerte es la de la Compañía, que tiene ciento ochenta brazas de altura, luego la de San Agustín, que tiene sesenta y dos; la otra de la Merced que pasa de ciento veintidós y la de la Catedral que tiene ciento cuatro". (Jouanen S.J. 2003: 2). Mario Cicala S.J. (1718-1790) escribió: “En cuanto al campanario de la iglesia está algo 


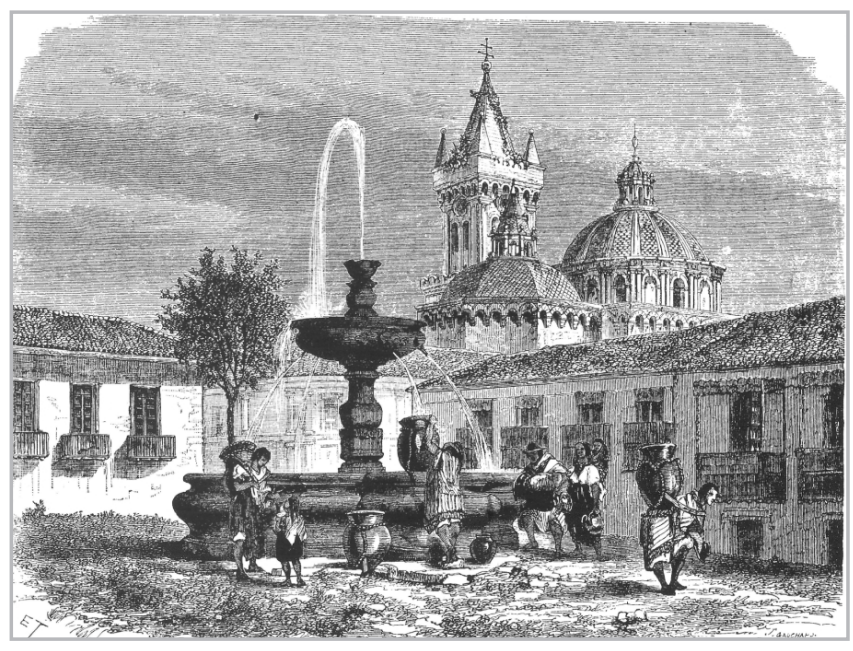

Figura 2.- Plaza y Fuente cerca de la Catedral. Dibujo E. Thérond según relato de Ernest Charton. 1860. Publicado en Le Tour du Monde, 401.

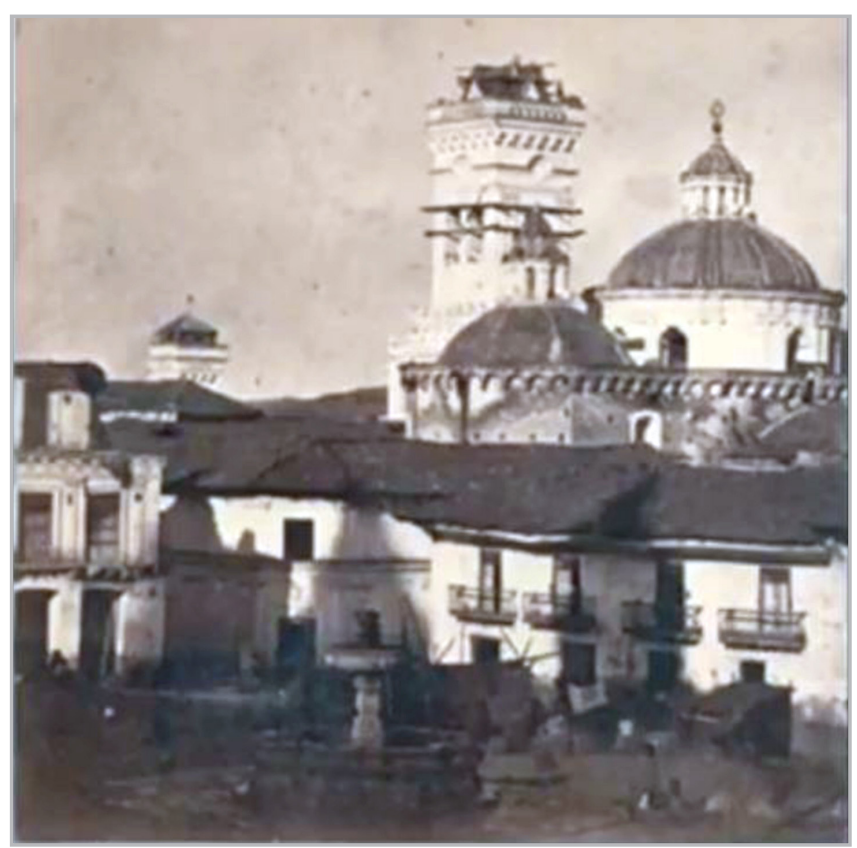

Figura 3.- Fotografía de la plaza de San Francisco y el conjunto jesuita. del Siglo XIX (Archivo FICJ - Anónimo)

separado de ésta, es muy alto, quizás sobrepasa a las demás... tiene tanta profundidad en los cimientos cuanta es la altura desde el pavimento hasta las campanas" (Cicala 1994:180).

De acuerdo con estas descripciones, la altura de las torres podría variar significativamente según las diferentes unidades de medida del siglo XVIII:

Braza española equivale a 1,6718 metros

Braza italiana equivale a 0,99635 metros

Braza de Milán equivale a 0,6481 metros

Braza del Estado Pontificio de Bolonia 0,635 metros

Braza de Nápoles 0,54216 metros

Tabla 01: OBREGÓN (1856): 34-37
La braza española en el año de 1726 equivale a dos varas o a 1,6718 metros según el sistema métrico decimal. Aplicada esta medida a la versión de Coleti, la altura de la torre de la Compañía de Jesús sería de 300,92 metros; imposible de construir en ese entonces, desproporcionada en relación al edificio y al perfil urbano de la ciudad.

La aplicación de la braza romana equivalente a 0,99635 metros daría como resultado una torre de 179,34 metros de altura; y la braza napolitana, que es la más pequeña encontrada en los sistemas métricos españoles e italianos, las 180 brazas equivalen a 97,588 metros, que tampoco guarda relación con la iglesia, la ciudad, la tecnología del momento, y tampoco con las fotografías y dibujos del siglo XIX. Por la revisión de las fuentes sobre el texto de Coleti, se infiere que su libro estaba destinado a maravillar a quienes lo leían; lo que desde el punto de vista científico le trajo más de un cuestionamiento, como la mención de una muralla en Mompox, en lugar de la albarrada construida junto al río para evitar las inundaciones (Giraldo 1952).

De allí que la estimación de la carta Annua y el texto de Kolberg sean referentes confiables para comprender que las torres de Quito perdieron altura. En el caso de La Compañía, la altura actual es de 23,40 metros. Según la carta Annua de 1642-1652 la torre tuvo 55 varas, es decir, 46,20 metros, dimensión cercana a las fotografías del siglo XIX que permiten deducir una altura similar. Sin embargo, en 1742 Charles Marie de La Condamine determinó que la torre de La Merced fue la más alta de la ciudad, sin dejar escrita la medida (De La Condamine 1986 Reedición: 135).

Con la imprenta, los tratados de arquitectura viajaron de Europa a América en las valijas de religiosos y alarifes como instrumento de apoyo al momento de decidir cómo construir un edificio. El orden, la proporción y la medida eran los referentes, la geometría el instrumento indispensable para la concepción del objeto. Su uso fue limitado entre personas relacionadas con la construcción. A manera de ejemplo, un tratado de Serlio que se encuentra en la biblioteca Nacional de Bogotá tiene escrito en su interior los nombres de cuatro dueños o usuarios, de los cuales dos de ellos aparecen como constructores en Quito, el primero es Alonso de Aguilar, maestro mayor y alarife de la obra de La Catedral; el segundo es Sebastián Dávila, quien dibujó lacerías para artesonados mudéjares en San Francisco (Gutiérrez 1977: 36). En ese entonces, un mismo libro podía circular por diferentes dueños y técnicos; era un documento especializado y de acceso restringido. De hecho, el convento de San Francisco tiene varios elementos tomados de los tratados de Serlio y Vignola que al parecer fueron los de mayor difusión.

\section{Síntesis comparativa de la geometría de la torre en relación con la iglesia.}

Este antecedente permite plantear la hipótesis de que las proporciones utilizadas en los tratados sirvieron de 
referencia en la gramática de la arquitectura americana y que las proporciones de la fachada podían determinar la altura de la torre de la Compañía. Para demostrar esta presunción, y sobre todo la relación geométrica y de proporción entre la fachada y la torre se compararon planos realizados en diferentes años: 1998, 2003 y 2019. En 1998 la Fundación Compañía de Jesús realizó dos estudios para conocer la altura aproximada de la torre de la iglesia para 1868. El primero fue a partir de análisis fotográfico, a cargo de Luis Subía, arquitecto de la fundación [Figura 4]; y el segundo a cargo de Inés del Pino, consultora independiente, quien utilizó el método geométrico para inferir la altura de la torre a partir de las proporciones de la iglesia [Figura 5]; para esto se utilizó el levantamiento planialtimétrico de la iglesia realizado por el Museo del Banco Central del Ecuador entre 1991 y 1992.

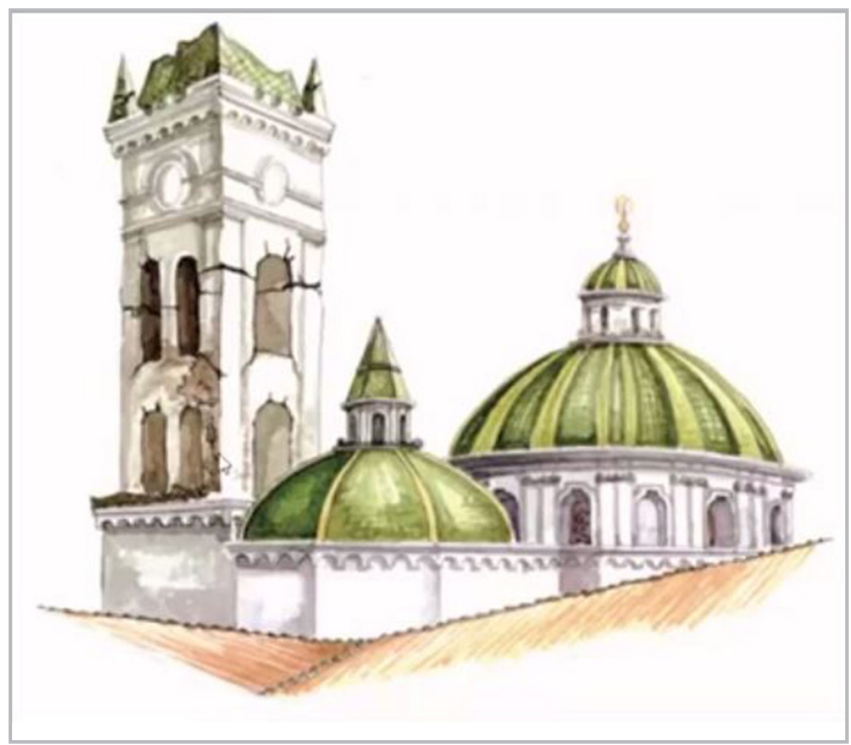

Figura 4.- Recreación del estado de la torre para el Siglo XVIII. Dibujo cedido por Luis Subía, 2005.

Posteriormente, en 2003 el Fondo de Salvamento de Quito (FONSAL), realizó un levantamiento de la fachada de la iglesia de la Compañía de Jesús de Quito mediante procesos fotogramétricos. El relevamiento de la información se hizo a partir de fotografía tradicional estereoscópica en 2D y a través de un procedimiento técnico se crearon imágenes en 3D que permiten visualizar relieves, rugosidades y fallas, facilitando la toma de datos sobre las superficies y reduciendo el tiempo de la producción gráfica.

En el último trimestre del 2019, mediante convenio entre la Universidad Internacional del Ecuador y el grupo de investigación de la Universidad de Sevilla (Innovación Tecnológica, Sistemas de Modelado 3D y Diagnosis Energética en Patrimonio y Edificación) se aplicaron técnicas de fotogrametría structure from motion (SFM) en la iglesia y como recurso principal el levantamiento geométrico mediante un escáner láser terrestre tridimensional (TLS) que realizó una captura masiva de datos (MDCS). Esta metodología se respalda en un modelo gráfico tridimensional que se obtiene de la toma real de medidas y geometrías del edificio histórico de manera digital no invasiva, la misma que fue registrada e integrada a una imagen $3 \mathrm{D}$ con base en un proyecto de modelado de información en edificios históricos (HBIM, Historic Building Information Modelling), una nueva concepción de información de edificaciones histórico-patrimoniales (Nieto 2021). En el caso de la iglesia la aplicación del método consiste en identificar las superficies sólidas como nubes de puntos (1497 millones de puntos) que son procesados mediante el sistema Cyclone Register 360 de Leica que realiza 70 escaneos del mismo objeto. El equipo utilizado fue BLK360, HDR (alta resolución), con este primer insumo del HBIM se sistematizó de manera integrada la información del edificio histórico, con el fin de proporcionar resultados de precisión del edificio patrimonial, precisión en cuanto a detalles arquitectónicos y decorativos, participación multidisciplinar de investigadores e información detallada para futuras intervenciones.

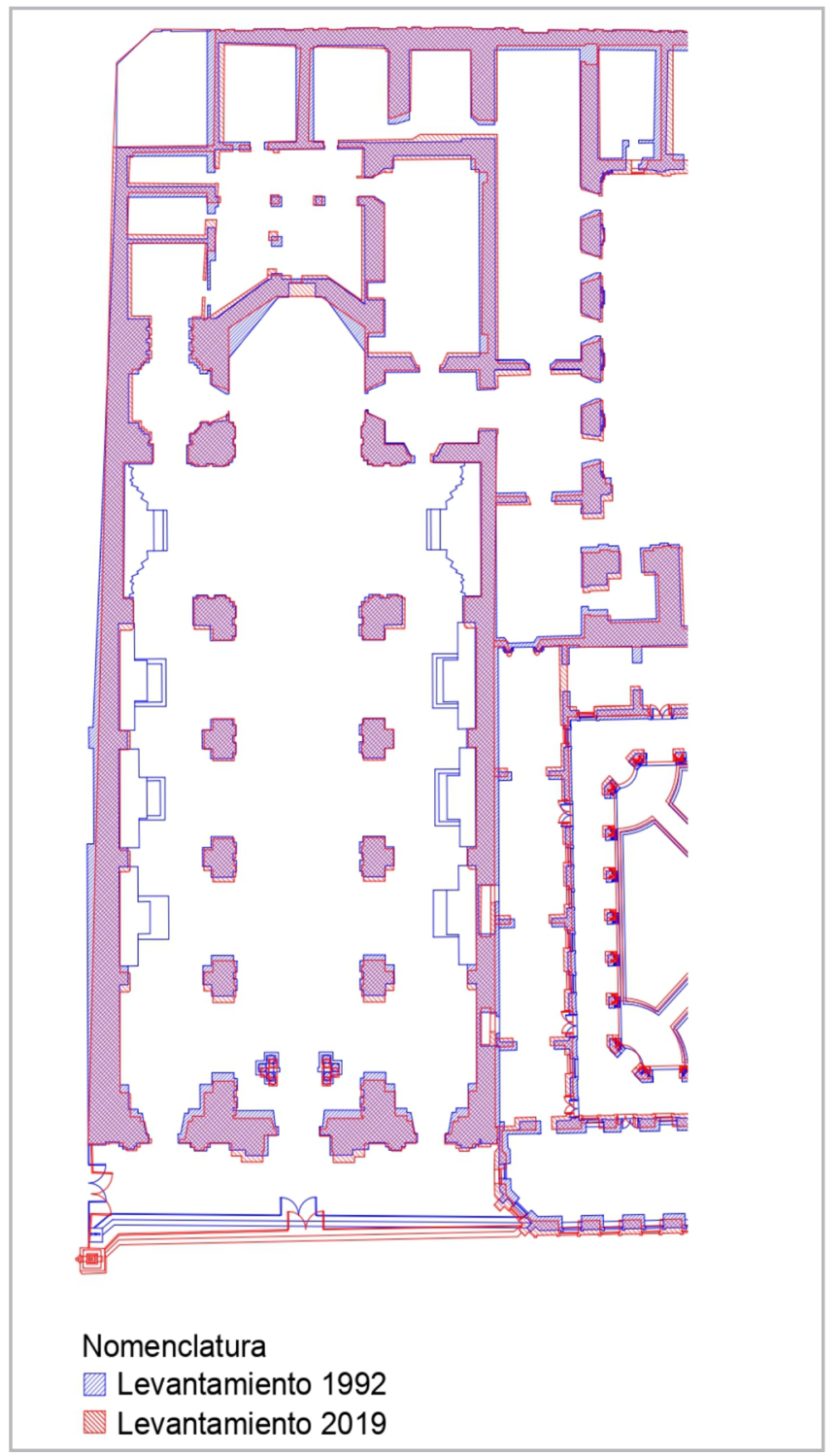

Figura 5.- Superposición de las plantas arquitectónicas de los levantamientos de 1992 y 2019 de la iglesia de la Compañía de Jesús. Elaboración propia. 
Como resultado de los levantamientos realizados en 1992 y 2019 existen algunas coincidencias en la iglesia que vale destacar: la primera es que al superponer las plantas de los dos levantamientos se observan variaciones menores en dimensiones [Figura 6] que no alteran la relación de proporciones geométricas generales: la relación entre la longitud y el ancho de la iglesia es 1:2, basada en una proporción aritmética de composición clásica. Las pilastras y muros se adaptan al predio irregular dando como resultado una planta asimétrica con variaciones mínimas entre los dos levantamientos. Los dos ingresos clausurados en el muro norte del plano de 1992 fueron insertados en el escaneo del 2019.

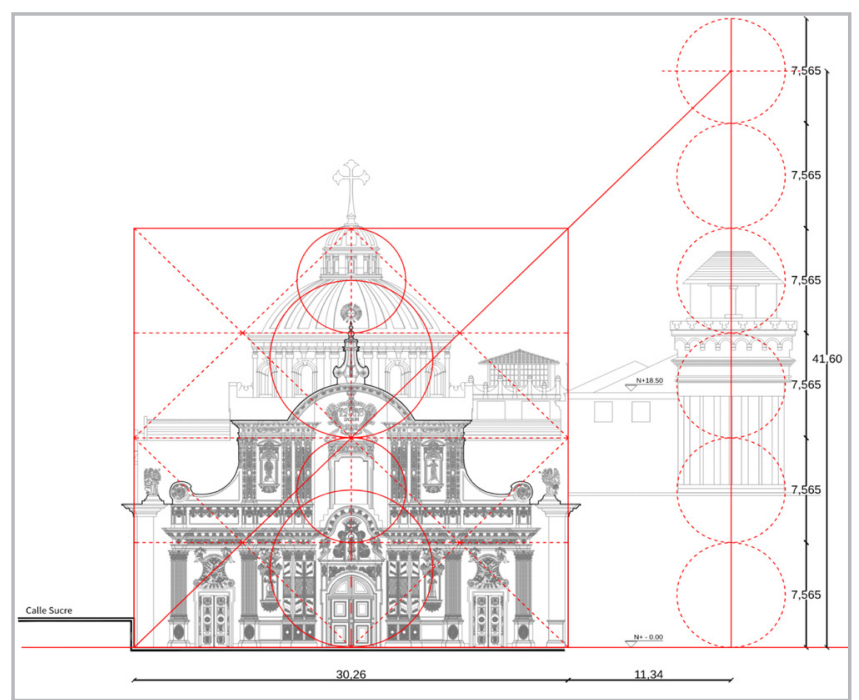

Figura 6.- Superposición de las plantas arquitectónicas de los levantamientos de 1992 y 2019 de la iglesia de la Compañía de Jesús. Elaboración propia.

La segunda coincidencia fue comprobar que la fachada, cúpula y cupulín de la iglesia se inscriben en un cuadrado de 30,26m. por lado [Figura 6]. La medida fue tomada en 2019 a ras del suelo -nivel 0,00 del ingreso-, mientras que el levantamiento de 1992 tomó el dato a la altura de 1,20 $\mathrm{m}$. desde el suelo, la diferencia de medidas entre los dos levantamientos es menor al 1\%. El cuadrado coincide con la clave del cupulín, sobre la cúpula mayor. En vertical, la fachada se divide en cuatro partes, cada una mide 7,565m., la primera: desde el piso hasta la cornisa del nicho de la virgen, sobre la puerta de ingreso. La segunda, hasta el arranque de la bóveda del templo. La tercera hasta la base de la cúpula mayor. La cuarta hasta la clave del cupulín. Al trazar las diagonales y los ejes de simetría del cuadrado, la intersección de líneas coincide en el corte arquitectónico con la base de la bóveda. Finalmente, al prolongar la diagonal del cuadrado, ésta se interseca con la línea del eje vertical del muro de la torre en la altura de 5,5 veces el módulo base de la fachada $(7,565 \mathrm{~m})$, es decir que la altura de la torre habría sido de 41,60 metros desde el nivel 0.00 de la iglesia [Figura 6].

La torre del campanario forma parte de los muros del antiguo colegio, se levanta a $+1,56 \mathrm{~m}$ con relación al ingreso de la iglesia, en un suelo de relleno. Por las variaciones topográficas,

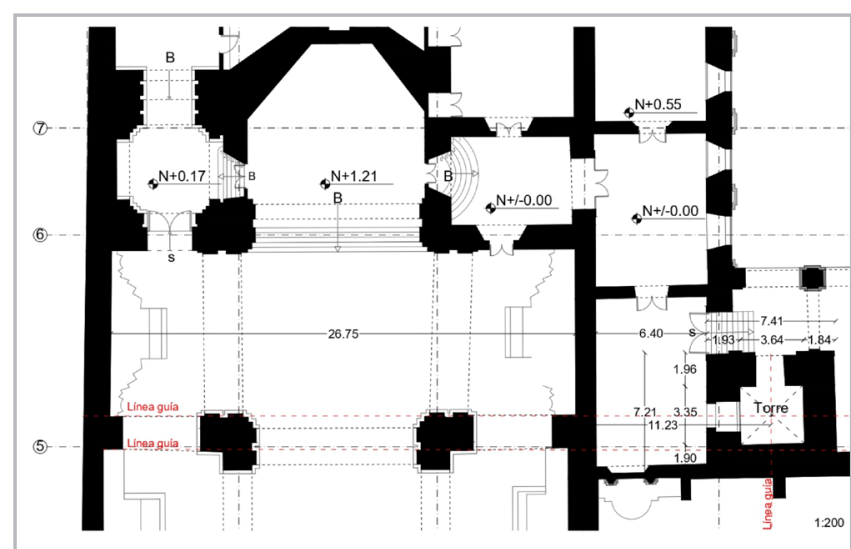

Figura 7.- Detalle de la torre insertada en la esquina del claustro y distancia con relación a la iglesia. Elaboración propia.

la altura de la torre sería de 40,04 m desde el piso del patio del claustro del colegio. Por otra parte, analizando las proporciones de la torre misma, su planta es casi un cuadrado de 7,21 x 7,41 m, dimensiones que se aproximan al módulo de $7,565 \mathrm{~m}$. En este sentido, la relación entre el ancho y el alto de la torre sería 1:6. Hay que observar que hasta una altura de aproximada de 10 metros la torre se halla embebida en los muros del claustro del colegio [ Figura 7].

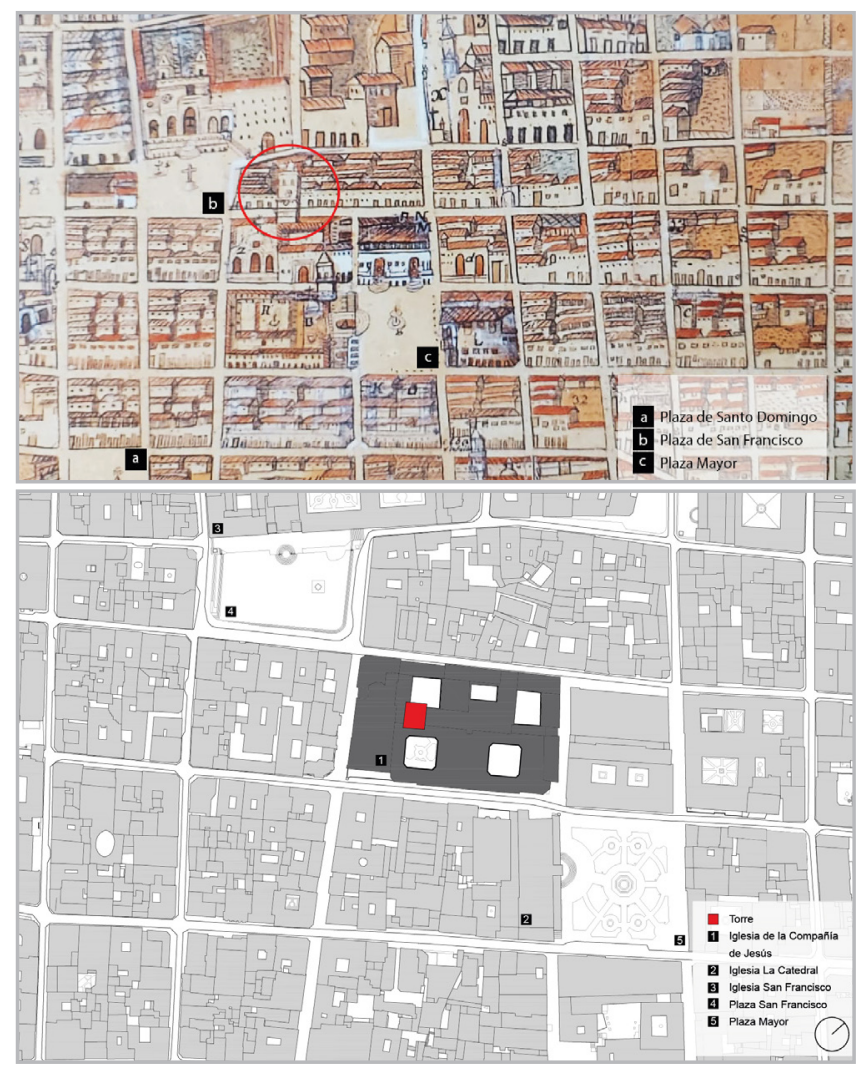

Figura 8.- La torre de la iglesia de la Compañía de Jesús en el contexto urbano de Quito. Izquierda: Detalle del plano de Quito de 1810 con la torre insertada dentro del predio y su relevancia visual desde las plazas principales de Quito: Plaza Mayor y San Francisco. Plano tridimensional, atribuido al Marqués de Selva Alegre, 1810. Sin escala. Reserva del Museo Municipal. Derecha: Detalle del plano catastral de Quito, 2020. Localización de la torre con relación al parque de la Independencia y la plaza de San Francisco. Quito, MDMQ. 
La torre no se levanta junto a la fachada, sino en el interior del predio, a la altura del presbiterio de la iglesia, en el claustro del colegio, y existe una separación de 6,40 metros desde el muro norte del templo al de la torre, en sentido horizontal [Figura 7]. Esta localización impide mirarla desde el pie de la fachada por la estrechez del atrio y la calle[8], limitación que fue percibida por fotógrafos y artistas del siglo XIX, quienes, para representarla tomaron distancia del edificio, a través de varios recursos visuales: la torre alineada y en diálogo con las de otros edificios religiosos: la Catedral y San Francisco, observada desde la plaza Mayor; otra vista frecuente es desde el atrio de la plaza de San Francisco[Figura 9], el campo visual abarca las cúpulas y torre de la Compañía, la torre de La Merced y la de la iglesia de San Juan en planos distintos que evidencian la perspectiva y la complejidad topográfica de la ciudad.

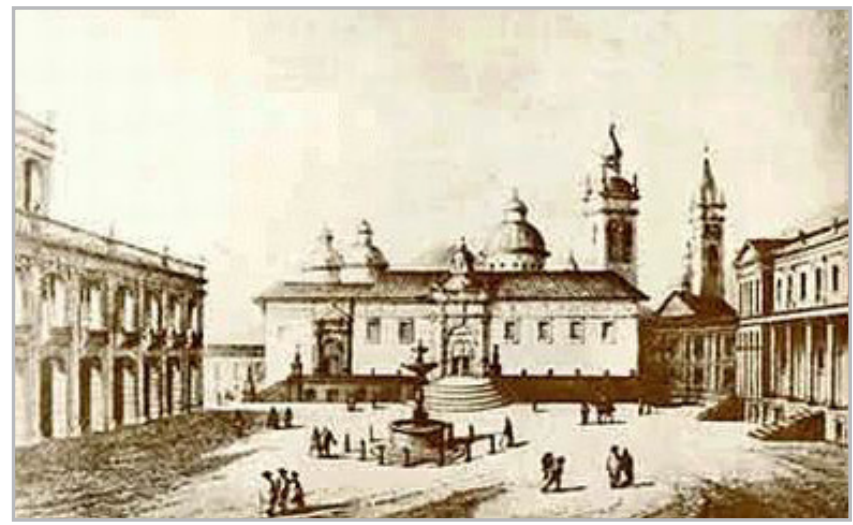

Figura 9.- Plaza Mayor de Quito, grabado. Publicado en "Relaçao de uma viagem a Venezuela, Nova Granada e Ecuador pelo conselheiro Lisboa", A. Lacroix, Verboeckhoven e CIA., Bruselas, 1866. (Foto Hirtz)

Existe un grabado de Miguel María Lisboa [Figura 9], diplomático brasileño que estuvo en Quito en 1853 con una misión de su país, que fue publicado en 1866. La imagen revela la intención de caracterizar a la ciudad y el perfil urbano desde la plaza Mayor de Quito mediante una secuencia de cúpulas y torres que habrían sido visibles con anterioridad al terremoto de 1868. En este caso, la imagen no se basa en fotografías, abarca un campo de visión que destaca las torres y cúpula de la Catedral y El Sagrario; las torres de San Francisco y la Compañía de Jesús.

Otro ejemplo es la pintura de autor anónimo: "La Plaza Mayor de Quito en Lunes Santo" proveniente del repositorio de arte del Banco de la República de Colombia en Bogotá [Figura 10]. En este caso, para encuadrar el motivo el artista toma distancia, se ubica sobre los edificios de la plaza para abarcar desde un mismo punto las principales torres de la ciudad: San Francisco con dos torres de dos cuerpos, la torre de la Compañía de Jesús, la torre de la Catedral; las cúpulas de la Catedral y El Sagrario. En el fondo destacan otras torres imaginadas; el conjunto sugiere el carácter de una ciudad señorial, una cantidad representativa de edificios religiosos y una sociedad que celebra uno de los rituales católicos más importantes del año: la semana Mayor.

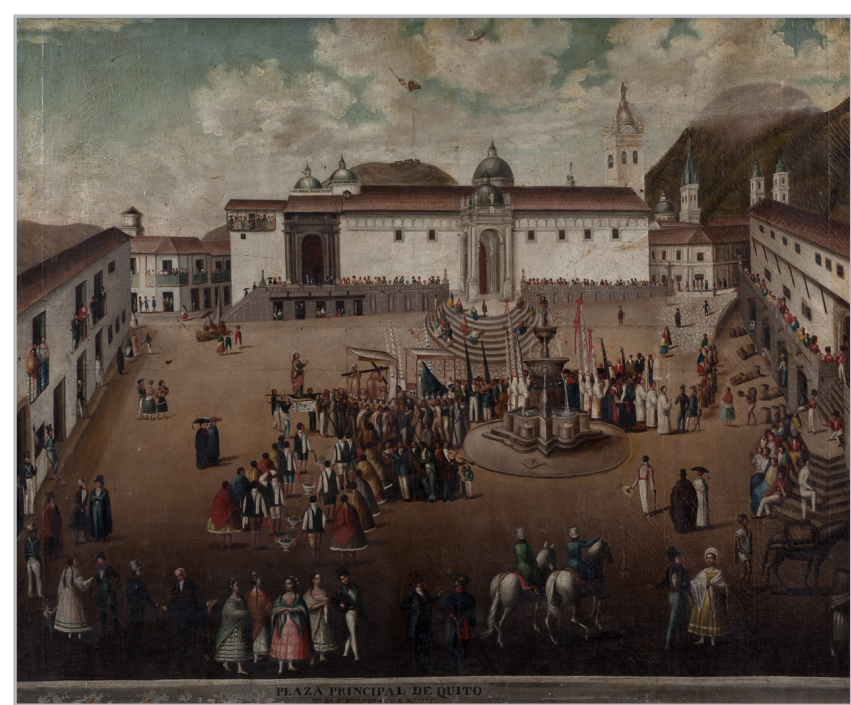

Figura 10.- Plaza Principal de Quito en Lunes Santo, Anónimo, 1800. óleo sobre lienzo. Registro: AP5172. Dimensiones: 64,2 x 81,8 cm. Banco de la República, Colombia.

De esta manera se puede inferir que la ubicación de la torre corresponde a un programa edilicio diferente al de las demás órdenes religiosas cuyas torres forman parte de la fachada. En el caso de Quito parecería que la intención fue crear un diálogo urbano entre edificios religiosos importantes, contribuir al carácter de la ciudad desde lo alto, es decir desde las colinas aledañas, en donde las 17 torres de las iglesias estructuran el perfil urbano, la quinta fachada de una ciudad católica y señorial. La observación de las torres desde las colinas que rodean la ciudad las convierte en hitos verticales, y dependiendo del punto de vista en que se encuentre el observador, se alinean de forma diferente. Esta visión permite valorar el conjunto con puntos de equilibrios cambiantes, relaciones de poder variables.

\section{Discusión y conclusiones}

El carácter de la arquitectura colonial de Quito está dado por la monumentalidad de los edificios religiosos y la relación armónica entre éstos, la arquitectura civil y espacios públicos en la larga duración. Los terremotos, erupciones volcánicas y las dificultades topográficas han sido determinantes en el acomodo de la traza y las proporciones de los edificios, por lo que es pertinente interrelacionar estas particularidades de la ciudad para valorar la arquitectura local.

Las ampliaciones o reformas en los edificios, y la destrucción por terremotos cambiaron la altura de las torres, pero el conjunto mantiene la escala y proporción. En la actualidad, las torres poseen las siguientes alturas: la torre de Santo Domingo, 41 metros; San Agustín, 40 metros; La Catedral tiene 38,78 metros; La Merced, 34,10 metros; la torre truncada de la Compañía de Jesús, 23,40 m. Por otra parte, la reconstrucción debió esperar, a manera de ejemplo, la reconstrucción de las torres de San Francisco concluyó el 
2 de febrero de 1893; la de San Agustín en 1910; Santo Domingo en 1924; La Merced en 1994, después del terremoto de 1987; y la Catedral en 1930. Es decir, el perfil de la ciudad es cambiante. Durante más de tres siglos estos elementos verticales fueron los hitos construidos de la ciudad, fueron referente de orientación, monumentalidad y poder de la iglesia.

Los levantamientos arquitectónicos realizados en el siglo XX, a más de la precisión, y la tecnología utilizada -fotogrametría terrestre o escaneo digital- permiten confirman la presunción del manejo de proporciones académicas utilizadas durante los siglos XVII al XIX como principio básico del equilibrio, proporción y belleza en la arquitectura en términos generales: La relación 1:2 utilizada en la configuración de la planta arquitectónica, o 1:6 para la altura de la torre. El aporte más destacado radica en la comprensión de la complejidad topográfica del sitio para lograr una unidad arquitectónica. En detalle, la torre es un elemento cuyas dimensiones varían en la base y en su desarrollo, no es paralela a la iglesia y tampoco se inserta en los ejes de composición del templo, hay ausencia de ángulos rectos en la planimetría. Las medidas adoptadas para este estudio son las tomadas en la base de la fachada y de la torre. Por otra parte, la base está embebida en los muros del claustro, lo que dificulta conocer el ancho del muro con precisión, en la parte que colinda con el centro cultural Metropolitano.

La relación proporcional de 1:6 en la torre de la iglesia de la Compañía de Jesús pone en contexto la descripción de la carta Annua y la de Joseph Kolberg cuyos criterios fueron referentes iniciales de este estudio. La comparación entre el levantamiento arquitectónico de 1992 y 2019 permite confrontar la descripción histórica con las medidas tomadas en el edificio, reconocer la aplicación de criterios de proporción y belleza presente en los tratados de arquitectura italianos no solo en planta y elevación, sino desde la perspectiva urbana, y las dificultades de su aplicación en la realidad topográfica de Quito, ya que la torre de la iglesia de la Compañía de Jesús es un elemento arquitectónico diseñado para ser observado mediante un recorrido y una sucesión de experiencias visuales: un hito llamativo que invita a la aproximación desde lejos, y de cerca, la sorpresa de la fachada que concluye con la maravilla del interior, un recurso que se inserta en el espíritu de lo barroco. Desde el exterior se encuentra en diálogo con las demás torres de la ciudad histórica y desde varias colinas que la circundan, quizás esto haya obligado a forzar la altura sobre los 40 metros con una solución tecnológica atrevida en su época. A esto vale añadir que Quito está rodeada de colinas y hasta el siglo XIX la observación de la ciudad desde lo alto fue uno de los atractivos para su gente en cuyas laderas identificaron sitios de privilegio para la observación denominados "miradores de pobres", desde aquí se identifica un perfil urbano cambiante según donde se sitúe el observador. Años más tarde, estos miradores fueron los lugares preferidos por los fotógrafos para realizar tomas atractivas de la ciudad.
El diseño de la torre sugiere una geometría y relación proporcional concebida en la segunda etapa de construcción del edificio a cargo del hermano Marcos Guerra, quien dio el carácter unitario a la arquitectura del conjunto jesuita utilizando recursos de perspectiva y decoración barroca que llaman la atención a quien visita el edificio. Uno de los aportes singulares de esta obra es el desplazamiento de la torre hacia el claustro del colegio, en el interior del predio [Figura 10]; esta decisión rompe con el diseño tradicional de fachada y torre sobre línea de fábrica, y es una pregunta que permanece: ¿por qué mantuvo la torre en el interior del predio? Una respuesta provisional es que la torre ya estuvo en ese lugar a su llegada. Una de las pruebas es que no tiene una proporcionalidad rigurosa que se observa en la volumetría de la iglesia, fachada y cúpulas. La torre no tiene ángulos de $90^{\circ}$ pero sus dimensiones se acercan a un cuadrado. El emplazamiento de la iglesia no es paralelo a la torre. El nivel de arranque de la fachada no es el mismo de la torre; no obstante, la solución constructiva resuelve la relación de proporción entre fachada y torre de manera visual y espacial antes que, desde la rigurosidad de la medida, se acomoda a la topografía y a la posibilidad de que destaque entre las demás cuando se la observa a la distancia y desde lo alto.

Este estudio permite valorar la capacidad de Marcos Guerra para componer y readecuar las piezas de un objeto arquitectónico preexistente al orden académico vigente, compatible con la naturaleza geológica de Quito, y una arquitectura con identidad propia y como una totalidad. Posteriormente, Jorge Vinterer puso el rigor en el orden de los elementos compositivos de la fachada, enlazando de manera visual a la torre. A esto se suma la calidad constructiva y estructural, y solidez del edificio, puesta a prueba en alrededor de un siglo de abandono y cinco terremotos que la han afectado hasta la actualidad, momento en el que tuvo el primer reforzamiento estructural.

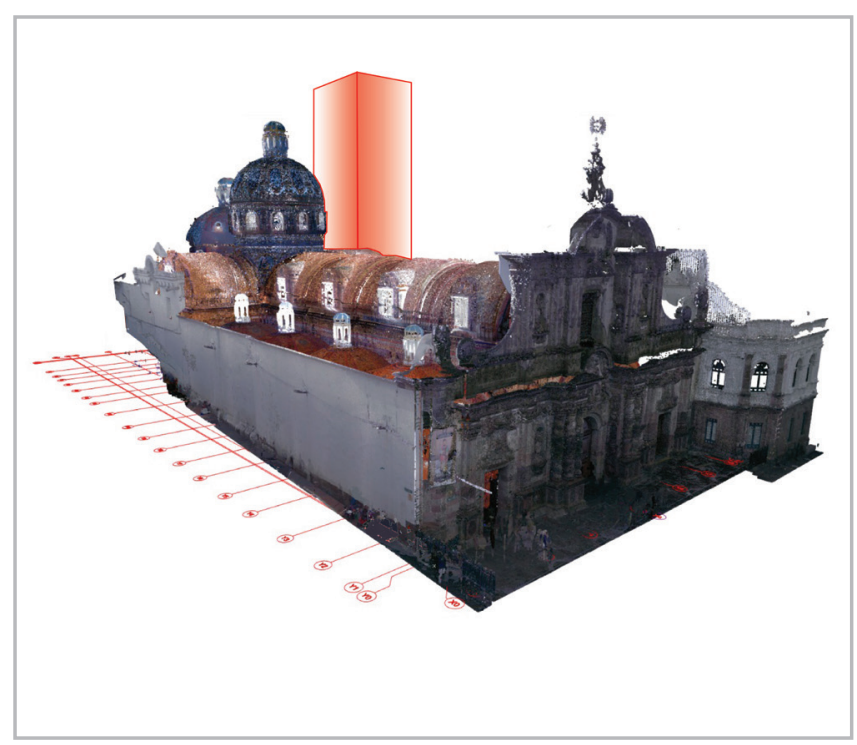

Figura 11.- Iglesia de la Compañía de Jesús según escaneo HBIM. Recreación de la volumetría de la torre. Elaboración propia 


\section{Agradecimientos}

Agradecemos la información proporcionada por la Fundación Compañía de Jesús: Diego Santander Gallardo y Luis Subía; a Christoph Hirtz por la fotografía [8], a Enrique Nieto de la Universidad de Sevilla por la información del levantamiento HBIM; a Alejandra Llumiluisa en la elaboración de los gráficos. El artículo es derivado del proyecto de investigación "Etapas constructivas de la arquitectura religiosa de Quito. Siglos XVIIXIX", auspiciado por la Pontificia Universidad Católica del Ecuador, Universidad Internacional del Ecuador UIDE, y Universidad Politécnica de Madrid.

\section{Notas}

[1] Expediente del plano de Dionisio Alcedo y Herrera. Quito, 132. N. 2. Archivo de Indias. Sevilla.

[2] Archivo histórico de la Sociedad Jesuita. Carta Annua desde los años 1642 hasta 1652 de la Provincia del Nuevo Reyno de Quito. Folio 25; Colegio de Quito, segunda parte de la Annua de la Provincia del Nuevo Reyno de Quito, fol. 25v.

\section{Referencias}

CICALA, M., (1994, reedición). Descripción histórico-topográfica de la Provincia de Quito de la Compañía de Jesús. Quito: Biblioteca Ecuatoriana Aurelio Espinosa Pólit, IGM.

DE LA CONDAMINE, C. M., (1986, reedición). Diario de viaje al Ecuador. Quito: Publitécnica.

DEL PINO, I. Y. H., (1990). Apuntes para una historia sísmica de Quito, Centro histórico de Quito. problemáticas y perspectivas. Quito: Municipio de Quito, Junta de Andalucía, 67-100.

EL NACIONAL (a), (1868). Obras Públicas. El Nacional, 19 agosto, 1.

EL NACIONAL (b), (1868). Obras Públicas. El Nacional, 10 septiembre, 1.

GIRALDO, A., (1952). El padre Juan Domingo Coleti y su Diccionario. Sociedad Geográfica de Colombia. (1). Año X, 1-20.

GUTIÉRREZ, R. y. G. V., (1977). San Francisco de Quito. Trama, 1(1): 36-38.

HERRERA, P., (1916). Apunte cronológico de las obras y trabajos del cabildo o municipalidad de Quito desde 1534 hasta 1714. Primera ed. Quito: Imprenta Municipal.

JOUANEN S.J., J., (2003). Historia de la Compañía de Jesús en la República del Ecuador. 1850-1950. Quito: PUCE.

KOLBERG, J., (1996). Hacia el Ecuador. Relatos de viaje. Quito: Abya Yala. NIETO, J. L. L. M. J., (2021). Implementation of a TeamWork HBIM for the Management and Sustainability of Architectural Heritage.
Sostenibilidad, 13 (4), 2161 https://doi.org/10.3390/su13042161

ORTIZ, A., (2008). Desde la primera piedra hasta la expulsión: 160 años de historia. En: Radiografía de la Piedra. Primera ed. Quito: FONSAL, 173-211.

RECIO S.J., B., (1947). Compendiosa relación de la cristiandad de Quito. Madrid: Instituto de Santo Toribio de Mogrovejo.

SALDARRIAGA, A. O. A., (2006). En busca de Thomas Reed. Arquitectura y política en el siglo XIX. Bogotá: Alcaldía Mayor de Bogotá.

SANTANDER, D. M. S. J. L., (2008). Un templo vivo. Sentido y misión de la restauración del templo y colegio de los jesuítas en Quito. En: Radiografía de la piedra. Los jesuítas y su templo en Quito. Quito: FONSAL, 309-337.

\section{Autor/es}

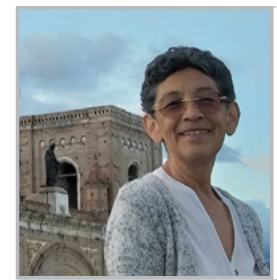

\section{Inés del Pino Martínez}

idelpinom@puce.edu.ec

Pontificia Universidad Católica del Ecuador https://orcid.org/0000-0003-4023-2271

Arquitecta. Doctora en Arte y Arquitectura por la Universidad Nacional de Colombia. Máster en Estudios de la Cultura por la Universidad Andina Simón Bolívar; Máster en Gobierno de la Ciudad con mención en Áreas Históricas. Profesora investigadora de la Pontificia Universidad Católica del Ecuador. Investiga temas relacionados con territorio y paisaje, arquitectura y ciudad ecuatoriana.

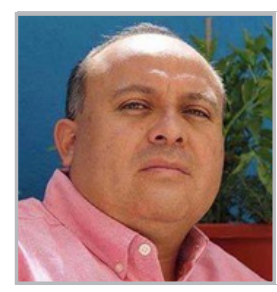

M. Lenin Lara Calderón

lenin.lara.calderon@alumnos.upm.es; mlara@uide.edu.ec

Universidad Politécnica de Madrid y Universidad Internacional del Ecuador https://orcid.org/0000-0002-8395-9932

Arquitecto, con formación investigativa, docente y profesional Máster en Restauración Arquitectónica y Máster Administración de Empresas Inmobiliarias. Estudiante de doctorado del Departamento de Construcción y Tecnología Arquitectónicas de la Universidad Politécnica de Madrid. Profesor investigador de la Universidad Internacional del Ecuador, ha colaborado en varios programas y proyectos de investigación sobre patología de la construcción y conservación del patrimonio.

Artículo enviado el 16/11/2021 Artículo aceptado el 19/01/2022 (c) $\underset{\mathrm{BY}}{\mathrm{N}} \$$ https://doi.org/10.37558/gec.v21i1.1080 